\title{
Education, income and happiness: panel evidence for the UK
}

\author{
Felix R. FitzRoy ${ }^{1}$ - Michael A. Nolan ${ }^{2}$ \\ Received: 11 January 2018 / Accepted: 30 October 2018 \\ (c) The Author(s) 2018
}

\begin{abstract}
Using panel data from the BHPS and its Understanding Society extension, we study life satisfaction (LS) and income over nearly two decades, for samples split by education, and age, to our knowledge for the first time. The highly educated went from lowest to highest LS, though their average income was always higher. In spite of rapid income growth up to 2008/2009, the less educated showed no rise in LS, while highly educated LS rose after the crash despite declining real income. In panel LS regressions with individual fixed effects, none of the income variables was significant for the highly educated.
\end{abstract}

Keywords Education · Income $\cdot$ Economic growth · Life satisfaction · Easterlin paradox

JEL Classification I31 $\cdot \mathrm{O} 47$

\section{Introduction}

Education is correlated with both income and health - each of which, in turn, has a positive effect on life satisfaction (LS). Those with higher education generally have access to more interesting and better-paid jobs, together with other non-pecuniary benefits. Meanwhile, manual labour is systematically correlated with lower LS - so it is not surprising that (higher) education is generally considered to be beneficial for subjective well-being, happiness or LS, as well as for objective individual economic

Electronic supplementary material The online version of this article (https://doi.org/10.1007/s00181018-1586-5) contains supplementary material, which is available to authorized users.

\footnotetext{
$凶 \quad$ Michael A. Nolan

M.A.Nolan@hull.ac.uk

1 School of Economics and Finance, University of St Andrews, St Andrews KY16 8QX, UK

2 Faculty of Business, Law and Politics, University of Hull, Hull HU6 7RX, UK
} 
and social goals. Thus, in their wide-ranging, cross-country survey of 'Happiness at Work' based on Gallup World Poll data, De Neve and Ward (2017) find a highly significant, positive effect of high education on LS in the presence of many other relevant controls such as health, income and employment - although a gender split for a much smaller sample based on European Social Survey data then indicates that a similar effect is only evident for men.

It is, therefore, initially rather surprising that a previous study of LS with British Household Panel Survey (BHPS) data found negative or insignificant effects of higher education in various specifications with numerous controls, while the positive effect was robust in German SOEP data (FitzRoy et al. 2014). However, using only Wave 1 BHPS data, Clark and Oswald (1996) report a negative relationship between a more specific job satisfaction variable and both education and comparison income. Via analysis of Wave 6-14 of the BHPS data, Powdthavee (2010) shows mainly negative estimates for education controls in pooled OLS estimation of LS and insignificant estimates for fixed-effects estimation. Green (2011) finds a negative effect of higher education on LS with Australian (HILDA) data using many controls, but Nikolaev and Rusakov (2016) find that higher education has a positive and increasing effect on LS from about the age of 35 in the same data set. Nikolaev (2016) also reports generally positive associations of education with various components of LS with the same data. Adding to conflicting results from HILDA, Powdthavee et al. (2015) estimate a structural model of education and life satisfaction and conclude that the direct effect of education is negative, while positive associations arise from the well-known positive effects of education on income and health. Overall, the existing literature contains mixed findings.

Here, we consider the UK over a rather longer time series. We extend the BHPS panel with the corresponding component of the Understanding Society data set (part of which involves individuals drawn from the BHPS) to study the development of life satisfaction (LS) and income across a couple of decades, in different education (and age) groups. Real household income (deflated by the Consumer Prices Index ${ }^{1}$ ) was always highest for the highly educated and for all groups grew substantially in the 10 years up to the financial crash of 2008/2009. The subsequent decline and partial recovery were steepest for the highly educated. That group also saw a rise in average household size around the time of the crash, whereas the households of the least educated tended to become smaller.

LS, on the other hand, rose fastest for the highly educated from a surprising lowest to being highest among the education groups, a significant increase over a period when the proportion of highly educated roughly doubled. LS declined steeply for the low education group up to and beyond the crash, in spite of their rising income. Overall, average LS declined pre-crash despite rapid income growth. Except for the highly educated before the crash, whose income and LS both increased, these results contradict standard economic growth theories, but are consistent with the Easterlin paradox found in macro data. Of course, mainly negative correlations of averages are no guarantee of the sign of the average of LS-income correlations at the individual

\footnotetext{
${ }^{1}$ Measured by ONS series D7BT.
} 
level-in general terms, this point can be traced back at least to Robinson (1950), and the coining of the term 'ecological fallacy' in Selvin (1958).

Various additional details emerge when we split the samples by age-specifically, for those aged under 45, and for those aged 45+. The LS of the younger, high education group overtook the rest by Wave 8 (1998/1999), while in the older group, LS only overtook the rest after the crash of 2008/2009 (in Wave 19), while relative incomes were similar. The older low educated suffered the steepest decline of LS over the whole period-from 5.40 (Wave 6) to 5.12 (Wave 23).

Easterlin's (1974) seminal paper found no correlation between long-term economic growth in rich countries, and subjective well-being (SWB-evaluated in surveys of LS or happiness). With 40 years of additional data, and economic growth, there is little evidence of any generally increasing SWB trend, ${ }^{2}$ (even in some of the fastest growing developing countries such as China). However, there is a strong cyclical relationship between real GDP per capita and SWB, with unemployment being a major cause of unhappiness that moves with the cycle, and critics have usually failed to distinguish carefully between trend growth and deviations from the trend (Easterlin 2013). Confirming and explaining these results, on the basis of 'loss-aversion', De Neve et al. (2014) show that economic downturns have negative effects on SWB which are several times the magnitude of the impact of longer periods of equivalent positive growth.

The paradox is deepened by the fact that richer people are generally happier than the poor in any one country at a given time, though many other factors such as health, family and employment are more important than income (but usually also correlated with income and education). ${ }^{3}$ The well-established importance of socio-economic status or relative income is often advanced as part of the explanation, but studies using only macro data on average happiness and per capita GDP obviously cannot explore this factor, while also omitting numerous important individual variables such as health, age and education, which do actually change in the aggregate over time. Other possible factors that could offset the benefits of growing average real incomes are rising inequality, reduced social mobility and the widely observed decline in many components of social capital, such as community, personal and family relationships, as well as security of employment-although these issues are beyond the scope of this paper. None of them seem to offer explanations for our surprising results. Adaptation to higher income was found to have only small effects by Layard et al. (2010).

It thus seems appropriate to use available large panel data sets, which follow individuals over time, to examine the effects of income (potentially, its level and growth) on their well-being, while controlling for both individual fixed effects and changing characteristics recorded in the survey data. Our main innovation here is to disaggregate the sample by three levels of education and by age. To the best of our knowledge, the education split in this context is a novel approach, which yields some really surprising results, including the lack of any significant own-or-comparison income effects on the LS of the highly educated, although their LS increased more than in other groups in

\footnotetext{
2 Helliwell et al. (2017).

3 Rich countries are also generally happier than poor countries, though there is much variation within these groups and possible problems with international comparisons of SWB which do not concern us here.
} 
the period. Another puzzle is why the high education group had lowest LS initially, but overtook the less educated to become most satisfied while higher education was rapidly expanding—see Blundell et al. (2016).

\section{Data and methodology}

Our main data are taken from Waves $6-10$ and $12-18$ of the British Household Panel Survey ${ }^{4}$ (BHPS), covering a period that runs from 1996/1997 to 2008/2009 (University of Essex, Institute for Social and Economic Research 2010) and from those parts of Waves 2-6 of the section of the new Understanding Society ${ }^{5}$ longitudinal study (Kantar Public, NatCen Social Research, University of Essex. Institute for Social and Economic Research 2016) that relate to active, consenting former members of the BHPS sample, covering a period ${ }^{6}$ from 2010-2011 to 2014-2015. An initial baseline of 214,704 observations is available, across the full income range. However, as is evident in Fig. 1, LS data were not collected for BHPS Wave 11 (17,609 observations for 2001/2002). Also evident from Fig. 1 (and its 95\% confidence limits) is the fact that not all of the time variation in average LS can be attributed to sampling variation. For regression analysis, we generate results for up to 178,382 observations across 23,748 individuals, with those cases where there are missing values, and the highest income outliers, ${ }^{7}$ excluded. As usual, we note the deliberate over-sampling of the smaller nations of the UK since Wave 9-so that about half of the individuals in the BHPS are from Scotland, Wales and Northern Ireland, ${ }^{8}$ compared with less than $20 \%$ in the overall population.

A plausible hypothesis is that those with higher education, who generally have the best-paid and most interesting jobs, would be most likely to enjoy increasing life satisfaction with higher incomes, so we split the sample into three groups. For the initial BHPS waves, classification through the International Standard Classification of Education (ISCED) is available — and the split is into higher (ISCED categories $5 \mathrm{a}$ and 6 - for first degrees and higher degrees), middle (ISCED categories $3 \mathrm{a}$ and $5 \mathrm{~b}$ - for higher secondary and middle/higher vocational) and low (ISCED categories primary, low secondary and $3 \mathrm{c}$-low secondary vocational) education. However, no

\footnotetext{
4 The earlier waves of the BHPS (up to Wave 10) were limited in coverage to Great Britain. The full UK (including Northern Ireland) is covered in Waves 12-18. BHPS data are available via the UK Data Service (formerly the UK Data Archive).

5 Since Wave 2 of Understanding Society is the first to follow on from BHPS Wave 18, we re-number the Understanding Society Waves (2-6) as 19-23.

6 With interviews taking place across calendar year boundaries (and two boundaries for Waves 21-22), a given Wave will see certain regressors defined according the year of interview, as appropriate to each individual.

7 A cut-off of 9.5 for the natural logarithm of (deflated) monthly household income is around $£ 160,000$ per year. This reduces the number of observations by 684, whilst a further 19 cases are excluded due to issues relating to the identification of individuals across waves. By definition, regressors measuring changes in household income between successive waves are not available for the first wave in which any individual responds. This is some 18,010 observations.

8 Across Waves 6-23, 44\% of observations are for individuals outside England. Northern Ireland was not included in the BHPS data until Wave 11 (2001/2002).
} 


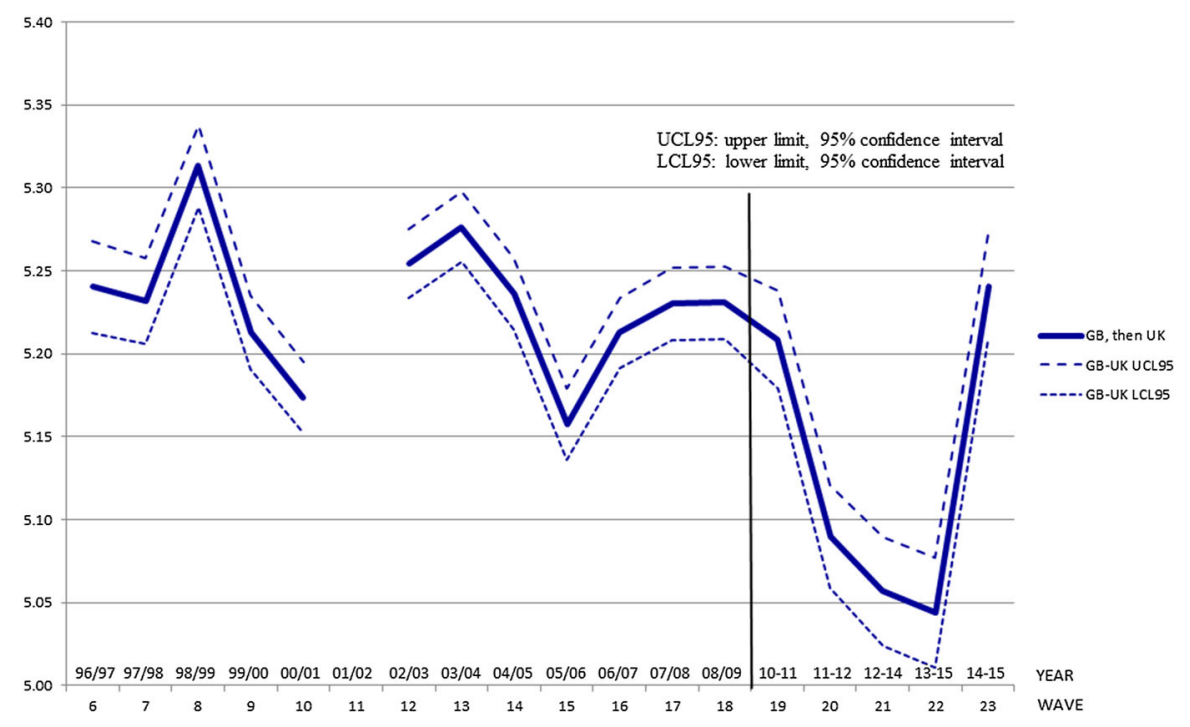

Fig. 1 Life satisfaction, BHPS and Understanding Society (USoc), Waves 6-10, 12-23

ISCED codings are yet available for the Understanding Society waves-so that the three-way split had to be undertaken on the basis of a less sophisticated derived highest qualification variable. ${ }^{9}$ Since the crucial difference is the striking and quite counterintuitive contrast between the higher and the two lower groups, we aggregate the latter pair to simplify Figs. 2, 3 and 4 (again, including confidence intervals) and our regressions.

Our estimation approach is quite similar to FitzRoy et al. (2014) —we use individual fixed effects in estimation of a LS equation with quite a number of controls-many of which are fairly standard when using BHPS data. These include marital status (including cohabiting), number of children, health status, education, labour market status, time spent in panel, whether year of last interview, log household size, age (via six age dummies to create seven age categories), housing ownership status, wave number and regions. We also tested the alternative of a traditional polynomial age specification — and found results quite similar to FitzRoy et al. (2014).

In Online Appendix, sample means are shown for many of the controls in Table A1a: the sample is also split by education level (see Fig. 5 as well). We also follow Moulton (1990) in recognising the potential (cluster related) effect of aggregate regressors on standard errors. Given that we are focusing on the estimation of individual-specific fixed-effects regressions, we assume clustering at the level of the individual.

For the crucial test of the effects of income on LS in different education groups, we include (deflated) own household income (for the month before interview) and comparison (peer group) income separately. The definition used here for comparison income follows that employed by FitzRoy et al. (2014)—whereby comparison groups are defined by age bands (between 3 years younger and 6 years older), sex, education

\footnotetext{
9 This split is essentially between degrees, A levels and GCSEs (alongside others, and none).
} 


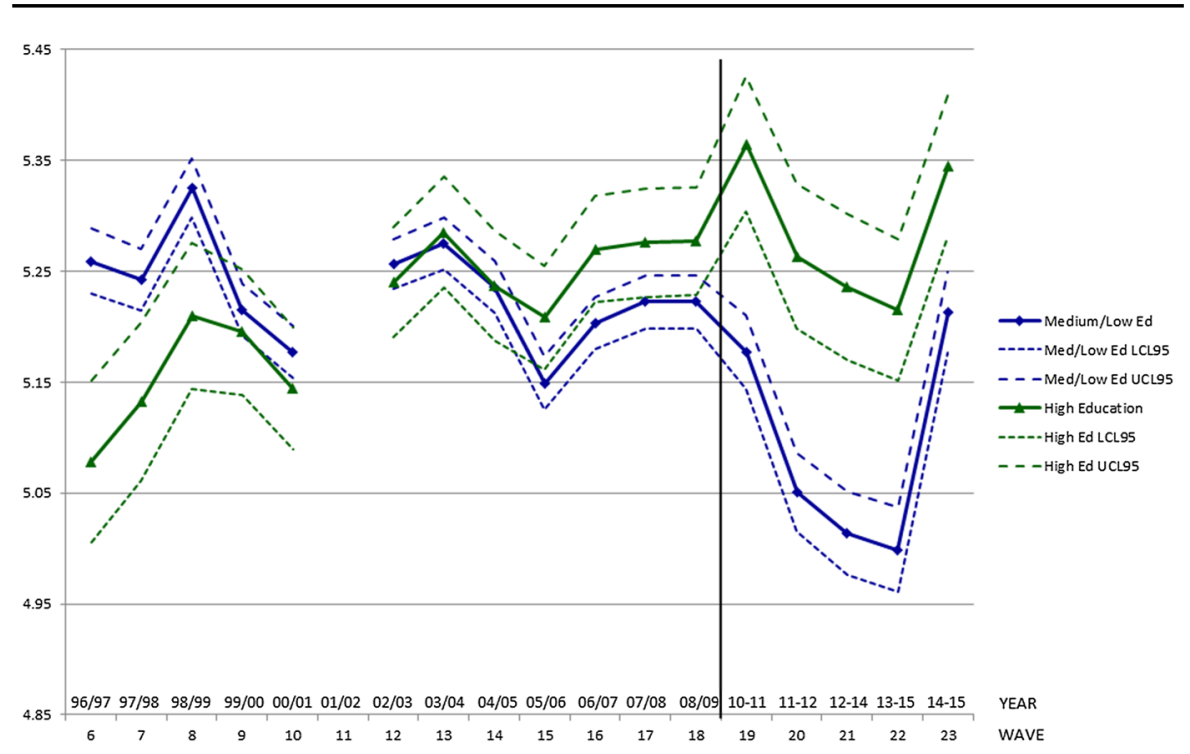

Fig. 2 Life satisfaction by education, BHPS and USoc, Waves 6-10 and 12-23

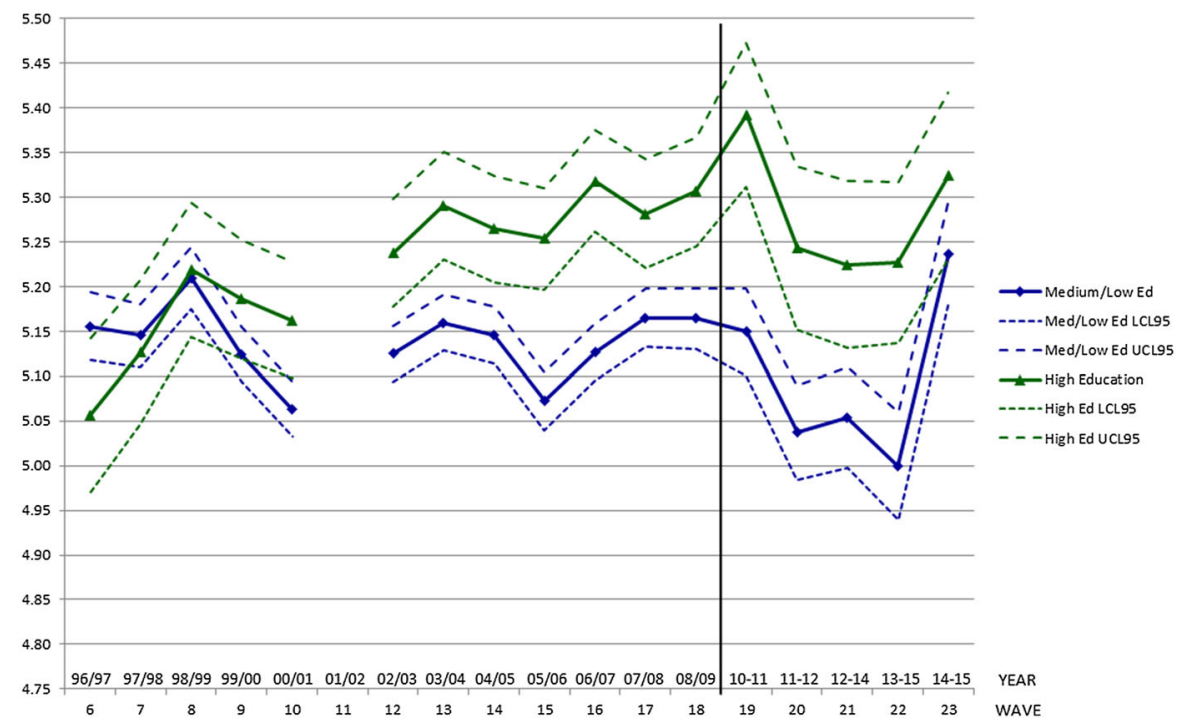

Fig. 3 Life satisfaction by education, BHPS and USoc, aged $<45$, Waves $6-10$ and 12-23

(two categories), region (three categories) and Wave. The groups are quite broad in specification - with a median cell size around 335 members, and a bottom decile at 80 members. We also experiment with the inclusion of upward and downward changes in own household income, allowing for asymmetric LS responses. In addition to including a full set of regional dummies (with Greater London as the reference region), we 


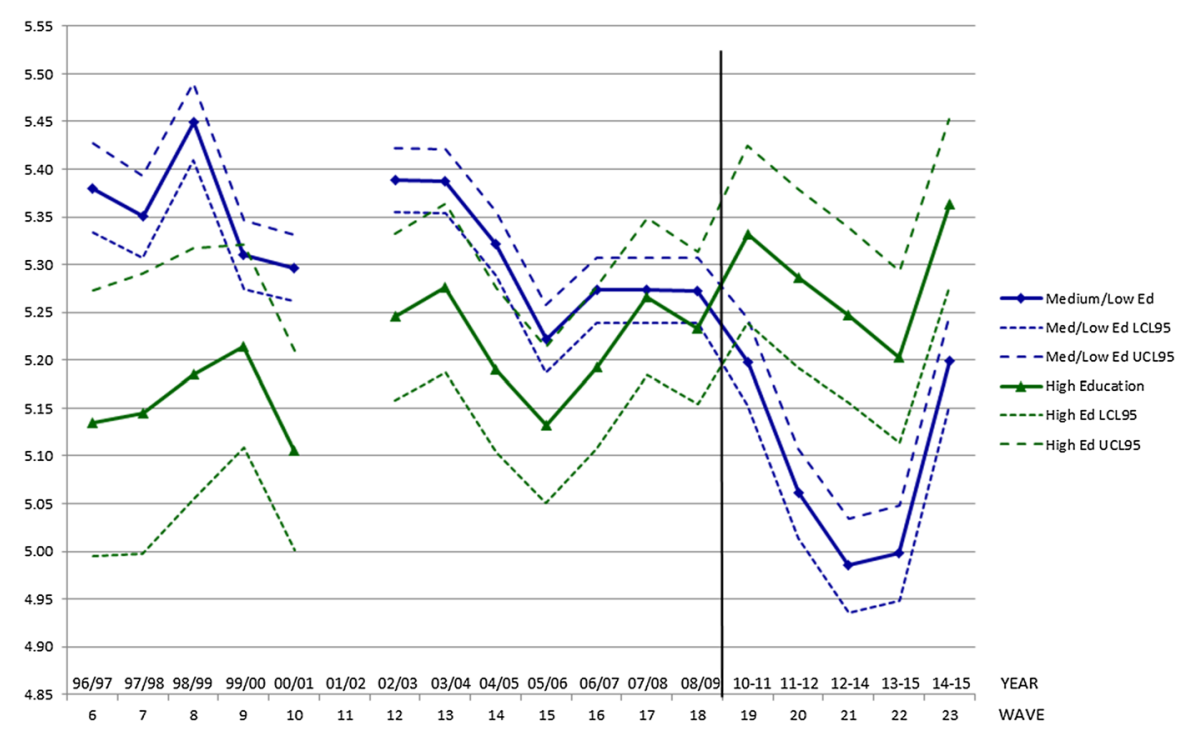

Fig. 4 Life satisfaction by education, BHPS and USoc, aged 45+, Waves 6-10 and 12-23

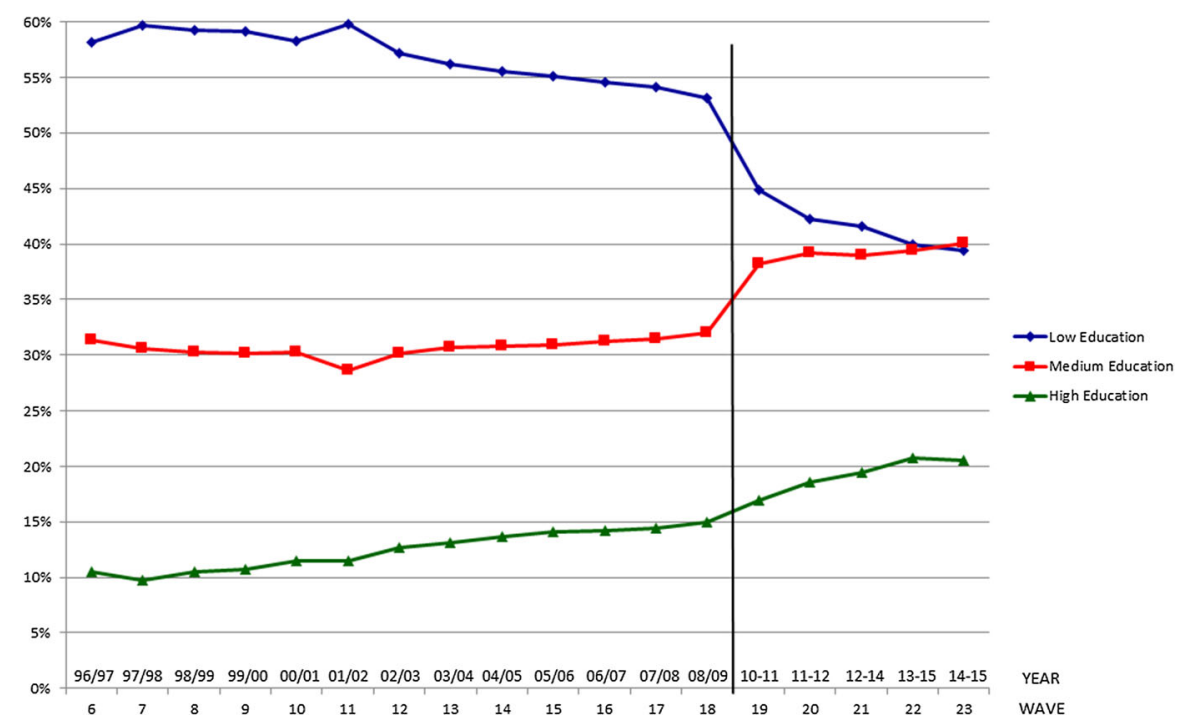

Fig. 5 Education categories, BHPS and USoc, Waves 6-23

control for (the ILO measure ${ }^{10}$ of) regional unemployment—which is not exclusively cyclical, of course-as well as regional house prices. ${ }^{11}$ The type of equation that is estimated-sometimes split by age range (under 45 and $45+$, respectively) and

\footnotetext{
${ }^{10}$ The annual ILO unemployment rates for NUTS1 regions of the UK are to be found in series YCNCYCNK and YCNM-YCNN.

11 The use of a simple average of house prices across all dwellings is a simplification, but it does enable the availability of a longer continuous run of data.
} 
sometimes split by education level (high vs. medium/low) — takes the following form, for the typical fixed-effects regression:

$$
\mathrm{LS}_{i t}=\beta_{0}+\beta_{1} \ln \bar{Y}_{j t}+\beta_{2} \ln Y_{i t}+\beta_{3} \ln \left(\frac{Y_{i t}}{Y_{i t-1}}\right)^{+}+\beta_{4} \ln \left(\frac{Y_{i t-1}}{Y_{i t}}\right)^{-}+\boldsymbol{\alpha} \boldsymbol{X}_{i t}+v_{i}+\varepsilon_{i t},
$$

where the $i$ subscript indexes the individual, the $t$ subscript indexes the wave (year) of the panel data and $j$ denotes the reference group (regarding individual $i$ ) for comparison income $(\bar{Y})$. Household income is denoted $Y$, while the + and - superscripts capture, respectively, the cases where deflated household income rises (relative to the previous wave) or falls. The separate terms allow, firstly, for a baseline effect of household income on life satisfaction. This effect is expected to be positive, and since income is entered in log form, the declining positive marginal utility of raw real household income is reflected naturally by a positive coefficient. In addition, any impact on current life satisfaction from wave-to-wave changes in household income (and, potentially, asymmetry in the respective impacts of a given magnitude of rise and fall in household income) can also be captured. The $\boldsymbol{X}$ term captures a vector of additional included controls, with an attendant vector of estimated coefficients $\boldsymbol{\alpha}$. The individual fixed effect is denoted $v$, while $\varepsilon$ is the remaining disturbance term.

We also tested for any additional effects of regional gross value added (GVA) per capita, in unreported regressions. It is clear from Fig. 10 (“Appendix 1") how different Greater London is, in this respect (as in many others) from the other UK NUTS1 regions. Like Pfaff and Hirata (2013), we found little systematic effect of regional GVA, which is not surprising given the inclusion of household income, and we also add comparison income. In contrast to their claims, this hardly supports Easterlin, since (on average) household incomes grow with macro income measures, and are closely related to LS in cross section, and in some of our panel results.

\section{Results and discussion}

Figure 1 demonstrates the lack of an obvious time trend in LS across Waves 6-18 of the BHPS ${ }^{12}$-although, within the Understanding Society waves, there appears to be some evidence of a lagged adverse reaction to the infamous Great Recession (itself evident via the real GVA per capita plots in Fig. 10). There is also a bounce-back in LS between Waves 22 and 23 .

We present plots of LS by education in Figs. 2, 3 and 4, log real household income in Fig. 6 and normalised ${ }^{13}$ real household income by education in Figs. 7, 8 and 9. The most surprising message from these plots is that the highly educated (top $14 \%$

\footnotetext{
12 Close examination of wave-specific means and standard errors for life satisfaction indicates a little more volatility than might be naturally expected, with high satisfaction in Wave 8 (1998/1999 — may be a sign of hopes springing from the 1997 General Election victory by Labour, after 18 years of Conservative governments), and low satisfaction in Waves 10 and 15 (2000/2001 and 2005/2006).

13 The normalising division by the square root of household size (the "square root scale") is employed in a number of OECD publications on income inequality and poverty (albeit across countries). In fact, the appearance of Figs. 7, 8 and 9 is similar to the look of corresponding plots for raw log real household
} 


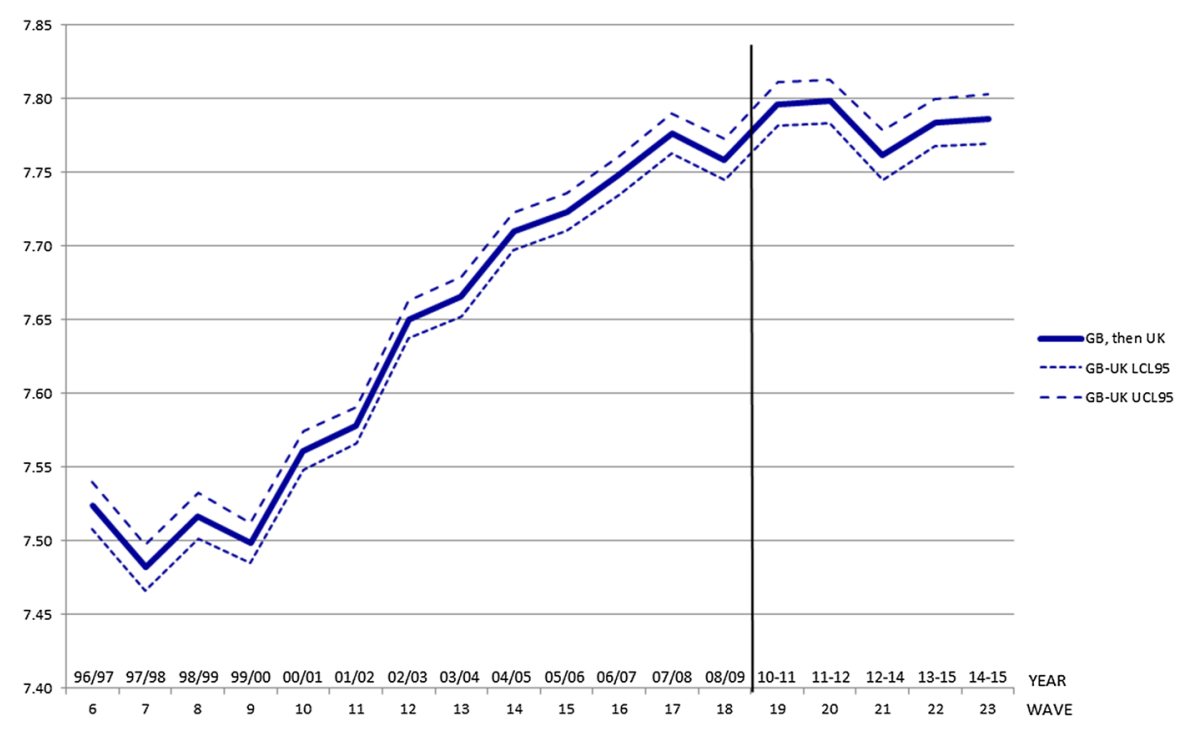

Fig. 6 Log income, BHPS and USoc, Waves 6-23

or so overall, but with a trend from $10 \%$ in Wave 6 to $15 \%$ in Wave 18 and $20 \%$ in Wave 23-as shown in Fig. 5) started with the lowest LS, but consistently have the highest LS from Wave 15 onwards (Fig. 2). This is despite the fact that the percentage growth (around 17\%) in their average real household incomes over the period was very similar to those with medium and low education $(17 \%$ and $18 \%$ ) and, at $14 \%$ for equivalised income, below that for those with medium and low education (18 and $21 \%$ ). An interesting further dimension is the expansion of the proportion of the UK population that are highly educated (see Fig. 5). Between BHPS Waves 6 and 18, this rose by $42 \%$ and $96 \%$ between BHPS Wave 6 and Wave 23 (Understanding Society Wave 6). ${ }^{14}$

Summary statistics are displayed for a few key variables in Table 1 - split by education level. Overall, there is a tendency towards a positive link between LS and education level. There is a more noticeable (and expected) positive link between household income and education level, and this unsurprisingly is also reflected in comparison income. The decline in average age by education level is consistent with the known ongoing increase in access to higher levels of education in the UK, over the last couple of decades (and beyond).

A further split of the sample into a younger group (those under 45 years) and an older group (those aged 45+) reveals that the positive relationship between LS and education

Footnote 13 continued

incomes. Figures 6, 7, 8 and 9 all include income data for BHPS Wave 11, although no LS data were collected for that wave.

14 The percentage increase among women over the same period was even greater, but we do not pursue the gender dimension further in this paper. It should be noted that there is noticeable attrition between Waves 6 and 23: differential attrition (by education level) might exaggerate the rise in the percentage of highly qualified. So too might the move away from an ISCED-based definition of qualifications in the Understanding Society data. 


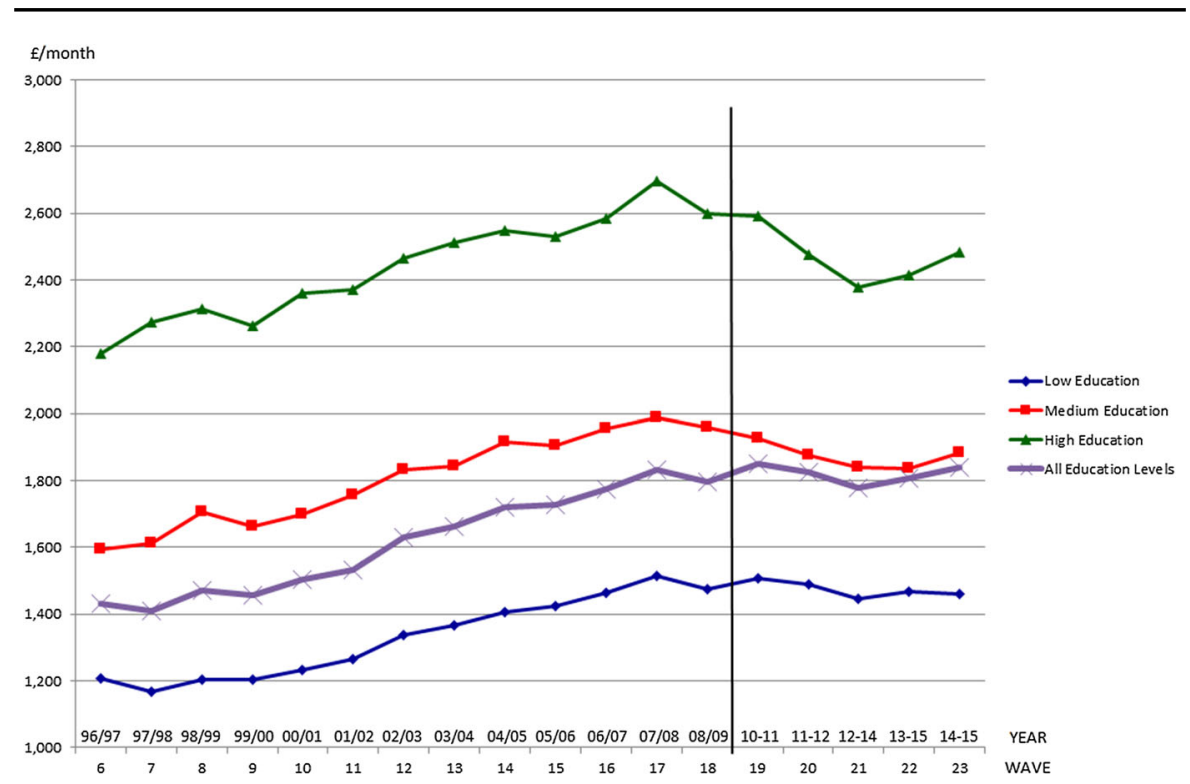

Fig. 7 Normalised income by education, BHPS and USoc, Waves 6-23

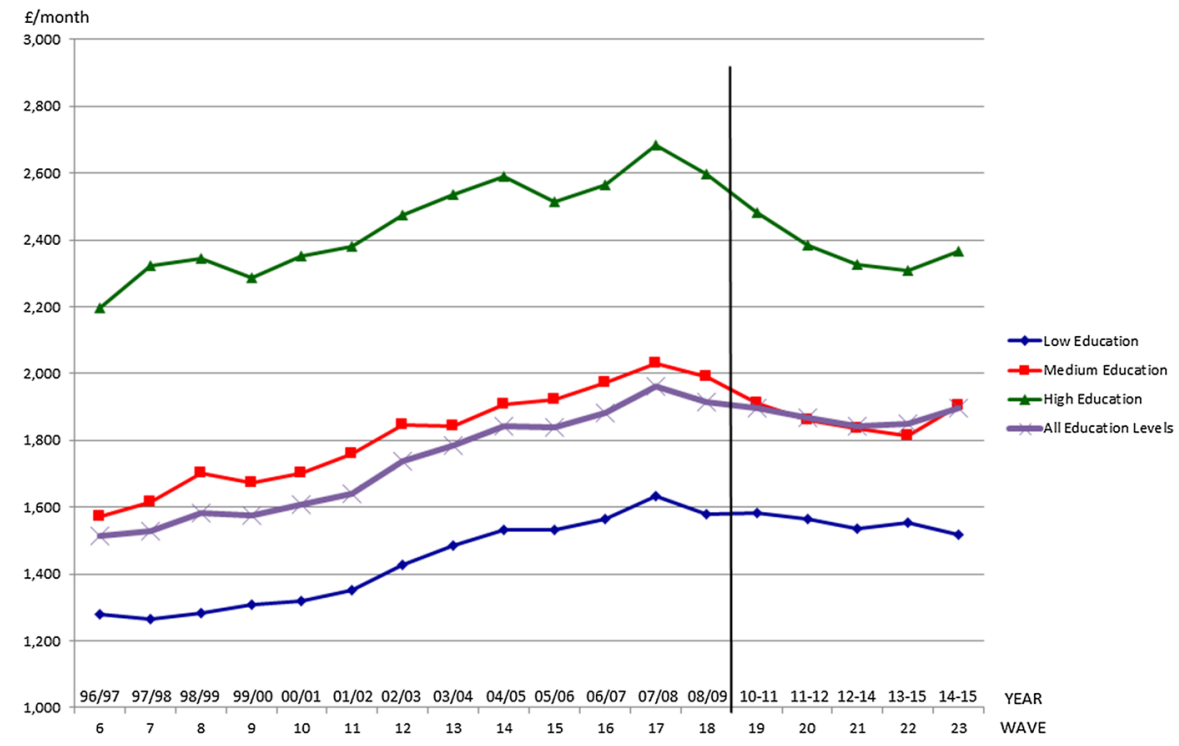

Fig. 8 Normalised income by education, BHPS and USoc, aged $<45$, Waves 6-23

level for the former is reversed for the latter. This can also be seen by examining Figs. 3 and 4, where the older highly educated have the lowest LS for most of the period. On the other hand, average household income (and also average comparison income) is robustly higher for an increase in education level, for both age groupings (see Tables 2 and 3; and also Figs. 8 and 9, on normalised income). For age itself within the younger group, the highly educated tend to be older-which is likely to be a reflection of the 


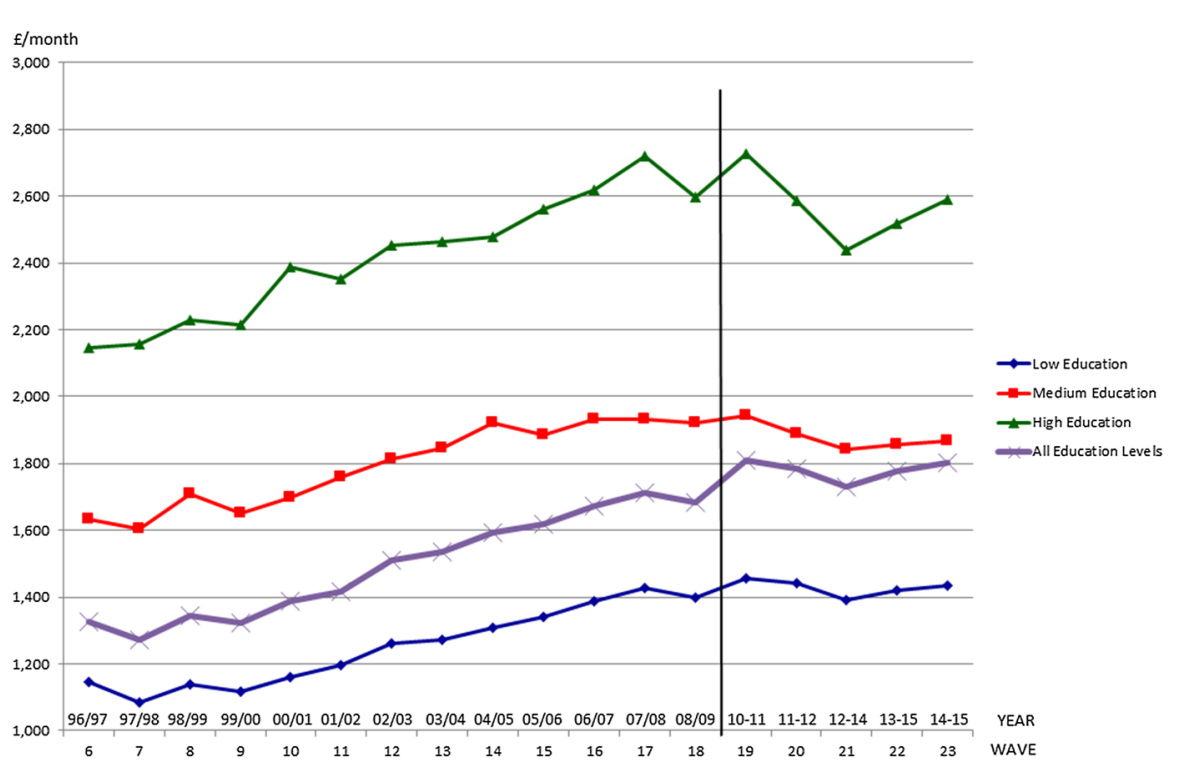

Fig. 9 Normalised income by education, BHPS and USoc, aged 45+, Waves 6-23

Table 1 UK, BHPS and USoc, Waves 6-10 and 12-23

\begin{tabular}{lllll}
\hline & $(1)$ & $(2)$ & $(3)$ & $(4)$ \\
& All & Low education & Medium education & \begin{tabular}{l} 
High education \\
\hline Summary statistics
\end{tabular} \\
Life satisfaction (mean) & 5.21 & 5.19 & 5.21 & \\
Life satisfaction (SD) & 1.34 & 1.42 & 1.27 & 5.24 \\
Age (mean) & 46.17 & 49.05 & 43.28 & 1.17 \\
Age (SD) & 18.46 & 19.54 & 17.48 & 41.94 \\
Household income (mean) & 2728.04 & 2248.48 & 3005.18 & 14.10 \\
Household income (SD) & 1836.48 & 1559.89 & 1838.08 & 3910.18 \\
Comparison income (mean) & 2751.80 & 2446.78 & 2697.91 & 4039.10 \\
Comparison income (SD) & 886.35 & 736.95 & 633.11 & 765.51 \\
Observations & 196,392 & 104,823 & 64,064 & 27,505 \\
\hline
\end{tabular}

longer time taken to complete education to a high level. Meanwhile, there is a negative relationship between age and education level within the older age grouping, which provides further evidence that the incidence of high education is increasing among successive birth cohorts. This view is broadly supported by Figs. 11 and 12, although a switch from low to medium education is noticeable for both age groupings. ${ }^{15}$

Most of the compositional aspects in the data set are unsurprising. For instance, the highly educated group is drawn disproportionately from Greater London, South East England and Scotland. Its members are more likely to be employed, and less likely to

$\overline{15}$ Alongside the move away from ISCED-based education groupings in the Understanding Society data. 
Table 2 UK, BHPS and USoc, Waves 6-10 and 12-23

\begin{tabular}{lllll}
\hline & $\begin{array}{l}(1) \\
\text { Under 45 years }\end{array}$ & $\begin{array}{l}(2) \\
\text { Low education, } \\
<45\end{array}$ & $\begin{array}{l}\text { (3) } \\
\text { Medium } \\
\text { education, }<45\end{array}$ & $\begin{array}{l}\text { (4) } \\
\text { High education, } \\
<45\end{array}$ \\
\hline $\begin{array}{l}\text { Summary statistics } \\
\text { Life satisfaction (mean) }\end{array}$ & 5.15 & & & \\
Life satisfaction (SD) & 1.27 & 5.08 & 5.18 & 5.25 \\
Age (mean) & 30.68 & 1.35 & 1.22 & 1.14 \\
Age (SD) & 30.22 & 30.35 & 32.64 \\
Household income (mean) & 3067.66 & 2644.16 & 8.33 & 6.68 \\
Household income (SD) & 1801.52 & 1576.07 & 1790.30 & 3980.88 \\
Comparison income (mean) & 3126.79 & 2908.18 & 2927.38 & 4142.67 \\
Comparison income (SD) & 639.15 & 403.20 & 412.20 & 579.63 \\
Observations & 98,002 & 45,272 & 35,934 & 16,796 \\
\hline
\end{tabular}

Table 3 UK, BHPS and USoc, Waves 6-10 and 12-23

\begin{tabular}{lllll}
\hline & $\begin{array}{l}(1) \\
\text { Aged 45+ }\end{array}$ & $\begin{array}{l}\text { Low education, } \\
45+\end{array}$ & $\begin{array}{l}\text { (3) } \\
\text { Medium } \\
\text { education, }\end{array}$ & $\begin{array}{l}\text { 45+ }+ \\
\text { High education, } \\
45+\end{array}$ \\
\hline Summary statistics & & & & \\
Life satisfaction (mean) & 5.26 & 5.28 & 5.25 & 5.23 \\
Life satisfaction (SD) & 1.41 & 1.47 & 1.33 & 1.22 \\
Age (mean) & 61.60 & 63.36 & 59.79 & 56.52 \\
Age (SD) & 11.54 & 11.71 & 10.99 & 9.61 \\
Household income (mean) & 2389.77 & 1947.68 & 2789.08 & 3799.29 \\
Household income (SD) & 1808.33 & 1478.25 & 1875.33 & 2272.10 \\
Comparison income (mean) & 2378.28 & 2096.01 & 2404.79 & 3878.33 \\
Comparison income (SD) & 938.97 & 739.93 & 736.64 & 967.24 \\
Observations & 98,390 & 59,551 & 28,130 & 10,709 \\
\hline
\end{tabular}

be unemployed or to be long-term sick or disabled (across the full age range and on both sides of the age split). They are also less likely to rent their dwelling. Among those aged $45+$, the highly educated are less likely to be retired and they enjoy a marked health advantage (present, to a lesser extent, in the younger age range too). Perhaps less obvious is the fact that the highly educated under 45 years have a lower average household size than the low or medium educated (maybe due in part to later marriage and starting of a family), but, among those aged $45+$, the highly educated have the highest average household size - possibly linked to a lower proportion having been widowed.

Our first estimation results are in Table 4, containing estimates of LS fixed-effects regressions across all education levels-initially across the entire age range, and then for younger $(<45)$ and older (45+) subgroups. Controls for high education are included (among the long list of controls), with an interaction to allow for a differential impact of high education on LS from Wave 14 (2004/2005) onwards (in line with Fig. 2). 
Table 4 Individual fixed effects, across education-UK, BHPS and USoc, Waves 6-10 and 12-23

\begin{tabular}{llll}
\hline Regressor & All & $<45$ & $45+$ \\
\hline Comparison income (log form) & $-0.171^{* * *}$ & 0.091 & $-0.307 * * *$ \\
Household income (log form) & $(-3.57)$ & $(1.07)$ & $(-4.80)$ \\
& $0.040^{* * *}$ & $0.063 * * *$ & 0.012 \\
Household income upward change & $(4.30)$ & $(5.03)$ & $(0.91)$ \\
& $0.023 * * *$ & $0.024 * *$ & 0.018 \\
Household income downward change size & $(3.14)$ & $(2.54)$ & $(1.64)$ \\
& -0.001 & 0.010 & -0.011 \\
Highly qualified $*$ Wave 14+ (interaction) & $(-0.07)$ & $(0.74)$ & $(-0.77)$ \\
Observations & $0.113 * * *$ & $0.106 * * *$ & $0.127 * * *$ \\
Individuals & $(6.03)$ & $(4.48)$ & $(3.96)$ \\
\end{tabular}

Dependent variable Life satisfaction. Controls for marital status (including cohabiting), number of children, health status, education, labour market status, time in panel, year of last interview, household size, age groupings, housing ownership, wave number and regions are included. Standard errors clustered at the individual level, robust t-statistics in parentheses

$* * * p<0.01 ; * * p<0.05 ; * p<0.1$

We report only coefficients of the various income variables, plus those for the high education * Wave 14+ interaction. A positive interaction effect is indeed evident for Waves 14-23, but the overall effect of being highly educated across those waves is significant at the $5 \%$ level only for the $45+$ age range. ${ }^{16}$ Own income and its upward changes have strong positive effects for the whole sample, and for those under 45 years taken alone. Meanwhile, comparison income has the positive, signalling effect for them that was found previously for those under 45 years ${ }^{17}$ _but the effect is statistically insignificant in our case. The usual negative effect for comparison income is again found for those aged $45+$, or for the whole sample across the entire age range. It should be noted that the number of individuals reported in the first column of Table 4, for the whole sample, cannot be expected to be the same as the sum of the totals in the other two columns (for the age split). This is because the observations for some individuals can be found on both sides of the age split boundary.

Although the alternative of pooled cross-sectional estimation is problematic for our unbalanced panel data, we have included Table A2a in Online Appendix, for additional context - with standard errors clustered this time by the comparison income grouping regressor. This shows similar comparison income results to those found previously in Table 8 of FitzRoy et al. (2014)—with a significant negative estimate for the full

\footnotetext{
16 Nikolaev and Rusakov (2016) find a positive effect of education on LS that increases with age in Australian panel data.

17 FitzRoy et al. (2014) put forward a 'hare and tortoise' model, as a plausible basis to explain a positive effect for comparison income amongst younger people (especially later developers, who can see higher incomes for their peers as a signal of the potential for their own future). Meanwhile, older people may tend to realise that they are unlikely to attain higher incomes of their peers-if they have already had many years of opportunity, with such incomes remaining unrealised.
} 
age range and also for the 45+ sample. Estimates for own household income are also broadly in line with that earlier work. ${ }^{18}$ Moreover, unreported regressions across the whole age range with comparison income interacted with the age grouping control categories and own income interacted with an 'aged 45+' dummy generated chiefly similar results to those in Table 24 of FitzRoy et al. (2014), both for fixed-effects and for pooled OLS.

In Table 5, we report the same specification for the highly educated, with the really remarkable result that none of the standard income variables is significant for either age group (or across the full age range). Recall, from Fig. 2, that wave-specific LS arithmetic means rose significantly between BHPS Wave 6 and BHPS Wave 18 for the highly educated, while the number of highly educated individuals rose by $50 \%$ (Fig. 5). Figure 3 indicates an increase in LS for the younger age group among the highly educated, but no significant change for the older age group. The impact of the Great Recession did seem to push down LS somewhat across Waves 20-22, albeit with a bounce-back in Wave 23. These trends in LS must be due to other factors. Of our controls, a few do have statistically significant attached estimates. For the full age range, as expected, economic activity status categories such as employee, self-employed, retirement, family care and full-time education are all positive for LS, relative to unemployment, and long-term sickness or disability is negative. Being married or cohabiting is positive for LS, compared with being single and never married. Good health and bad health each have the expected impact on LS, compared with the baseline health category. Sampling variation may be a component in explaining the insignificance of income regressors (especially given that this education grouping contains fewer observations). In an effort to investigate the importance of low statistical power for our findings, a similar fixed-effects regression specification was estimated with the log of real household income as the dependent variable. This generated quite a few more statistically significant estimates-and overall goodness of fit measures around 5-7 times greater. The pooled cross-sectional results for this group (Table A2b) do not appear to offer solutions to this puzzle: instead, alongside some standard positive effects for own household income level, some additional queries are raised-by the significant negative estimate for comparison income among those under 45 years, and also the positive estimates for the magnitude of negative changes in own income.

For medium and low education (Table 6), the effect of comparison income on LS is almost fully consistent with FitzRoy et al. (2014)—although only statistically significant at the $10 \%$ level (and still negative) for the younger age range. However, own income and upward changes are only positive and significant for those aged under 45. For this stratum of education, the older group sees the only substantial prerecession decline in LS (Fig. 4). Both age groups have rising (normalised) real income, pre-recession (Figs. 8 and 9). Table 6 shows a negative effect of comparison income for the $45+$ group, and that - together with the rising real income trend-could be part of the explanation for a pre-recession fall in LS. The offsetting negative externalities of economic growth, and associated social change such as increasing prevalence of nonstandard and precarious employment, would instead be expected to impact especially

\footnotetext{
18 However, that earlier work did not have additional regressors for upward and downward changes in own income.
} 
Table 5 Individual fixed effects, high education-UK, BHPS and USoc, Waves 6-10 and 12-23

\begin{tabular}{llll}
\hline Regressor & All & $<45$ & $45+$ \\
\hline Comparison income (log form) & -0.038 & -0.020 & 0.031 \\
& $(-0.41)$ & $(-0.13)$ & $(0.25)$ \\
Household income (log form) & 0.016 & 0.043 & -0.022 \\
& $(0.69)$ & $(1.53)$ & $(-0.60)$ \\
Household income upward change & 0.016 & 0.008 & 0.031 \\
& $(1.05)$ & $(0.46)$ & $(1.14)$ \\
Household income downward change size & 0.010 & 0.048 & -0.033 \\
& $(0.38)$ & $(1.46)$ & $(-0.84)$ \\
Highly qualified * Wave 14+ (interaction) & 0.028 & -0.243 & 0.403 \\
& $(0.18)$ & $(-1.12)$ & $(1.41)$ \\
Observations & 25,514 & 15,228 & 10,286 \\
Individuals & 3,427 & 2686 & 1419
\end{tabular}

Dependent variable Life satisfaction. Controls as in Table 4, except for education $* * * p<0.01 ; * * p<0.05 ; * p<0.1$

Table 6 Individual fixed effects, medium/low education-UK, BHPS and USoc, Waves 6-10 and 12-23

\begin{tabular}{llll}
\hline Regressor & All & $<45$ & $45+$ \\
\hline Comparison income (log form) & $-0.200^{* * *}$ & $0.228^{*}$ & $-0.362^{* * *}$ \\
& $(-3.41)$ & $(1.93)$ & $(-4.90)$ \\
Household income (log form) & $0.041^{* * *}$ & $0.060^{* * *}$ & 0.019 \\
& $(4.02)$ & $(4.19)$ & $(1.30)$ \\
Household income upward change & $0.025^{* * *}$ & $0.031^{* * *}$ & 0.014 \\
& $(2.90)$ & $(2.66)$ & $(1.15)$ \\
Household income downward change size & -0.006 & -0.001 & -0.009 \\
& $(-0.51)$ & $(-0.04)$ & $(-0.59)$ \\
Observations & 152,868 & 70,122 & 82,746 \\
Individuals & 21,390 & 13,015 & 11,307 \\
\hline
\end{tabular}

Dependent variable Life satisfaction. Controls as in Table 4, except for education (medium dummy only) $* * * p<0.01 ; * * p<0.05 ; * p<0.1$

on the younger less qualified persons. ${ }^{19}$ Corresponding pooled cross-sectional results (Table A2c) also appear similar to their counterparts for the full sample across the whole education range-albeit now with a statistically significant positive effect of comparison income on LS, for those aged under 45.

Given especially our struggle to explain life satisfaction for the highly educated, we consider the potential for a role of the Big Five personality traits, and especially neuroticism - as examined by Proto and Rustichini (2015), and found to 'mediate the effect of income on life satisfaction'. The UK part of their work uses the BHPS data.

\footnotetext{
19 Unfortunately, such trends are not picked up by means of the standard labour market controls included within our regression specifications.
} 
However, one practical difficulty is that only BHPS Wave 15 (2005/2006) includes Big Five data-as also used in the household finances context by Brown and Taylor (2014). Proto and Rustichini (2015) argue_-quite plausibly — that such traits are very stable for most people across much of their lifespan. However, fixed-effects estimation could not use raw Big Five scores taken from a single wave. Much of the analysis in Proto and Rustichini (2015) is based on the interaction between (standardised) Big Five scores and a quadratic function of income, and they also include random-effects estimation. For our investigations, the assumed zero correlation between the disturbances and the regressors, inherent in random-effects estimation, appears to be a binding (and distorting) constraint. Also, since Understanding Society Wave 3 (Wave 20 within our composite panel, for 2012-2014) also includes Big Five data - as used by Brown and Taylor (2015) to examine charitable giving behaviour - we also incorporated that information.

Although we standardise neuroticism scores prior to regression estimation, we do not undertake a preliminary regression to generate residuals as a replacement for the standardised scores, to net out certain systematic effects. This is largely because Proto and Rustichini (2015) find little difference in the results on such a basis. Our results are given in Table 7-to compare with the respective left-hand columns of Tables 4, 5 and 6. In each instance, estimates from previously listed regressors remain very similar. However, in two out of three instances, the income-neuroticism interaction's estimates are statistically significant at the 5\% level. Although it is for the highly qualified that the estimate is insignificant, its similar magnitude at least suggests the possibility that insignificance may be linked to the smaller sample size. Meanwhile, the neuroticism score estimates are significant and negative in all three columns of Table 7. Overall, it seems that the income-personality trait interaction may offer useful additional evidence, although it still appears that the picture is less clear for highly educated individuals than for others (see Online Appendix Tables A2di-A2diii for full sets of regression results). Of course, this impression is emphasised by our (wellfounded) primary concentration on fixed-effects estimation-rather than pooled OLS or random effects, both of which generate greater statistical significance for more of the included regressors. ${ }^{20}$ Note that our specifications including the Big Five have a systematic linkage with BHPS attrition-in the sense that any individual who left the panel prior to Wave 15 (and had not returned by Wave 23) cannot have a score for any of the Big Five personality traits. ${ }^{21}$

Thus, in one sense we disagree with Easterlin (2013) by finding rising household incomes and LS for the high education group up to the recession, but we are consistent with his paradox for the less (low/medium) educated - since LS declined over this period in spite of faster rising income. Our fixed-effects estimation highlights a major puzzle - the almost complete lack of significance of any of the income variables in explaining rising LS for the high education sample. With the expansion of UK higher

\footnotetext{
20 Indeed, unreported results for specifications to measure "between" effects-essentially using the individual's across-time mean of each variable to focus on cross-sectional variation-demonstrate statistically significant coefficients for logged own income, and almost always also its interaction with neuroticism, for the various groupings in Tables 4, 5 and 6.

21 A (neuroticism) missing value dummy was included, as a control. Its attached estimate was negative but insignificant under fixed-effects estimation-and of the same sign and greater significance for random effects, and for pooled OLS estimation of the highly educated sub-sample.
} 
Table 7 Individual fixed effects, whole age range-UK, BHPS and USoc, Waves 6-10 and 12-23

\begin{tabular}{llll}
\hline Regressor & All & High education & Medium/low education \\
\hline Comparison income (log form) & $-0.171^{* * *}$ & -0.033 & $-0.200^{* * *}$ \\
& $(-3.56)$ & $(-0.36)$ & $(-3.41)$ \\
Household income (log form) & $0.040^{* * *}$ & 0.018 & $0.041^{* * *}$ \\
& $(4.38)$ & $(0.75)$ & $(4.09)$ \\
Household income (log form)* neuroticism & $0.016^{* *}$ & 0.024 & $0.016^{* *}$ \\
& $(2.45)$ & $(1.48)$ & $(2.23)$ \\
Household income upward change & $0.023^{* * *}$ & 0.016 & $0.024^{* * *}$ \\
& $(3.10)$ & $(1.04)$ & $(2.88)$ \\
Household income downward change size & 0.00004 & 0.012 & -0.005 \\
& $(0.004)$ & $(0.45)$ & $(-0.43)$ \\
Highly qualified * Wave 14+ (interaction) & $0.112^{* * *}$ & 0.031 & \\
& $(6.00)$ & $(0.19)$ & \\
Big five neuroticism (standardised) & $-0.194 * * *$ & $-0.280^{* *}$ & $-0.190^{* * *}$ \\
Observations & $(-3.70)$ & $(-2.12)$ & $(3.30)$ \\
Individuals & 178,382 & 25,514 & 152,868 \\
\hline
\end{tabular}

Dependent variable Life satisfaction. Controls as in Tables 4, 5 and 6 (respectively), plus a control dummy for missing data on neuroticism

$* * * p<0.01 ; * * p<0.05 ; * p<0.1$

education reaching more families without any prior tradition, it might be that beneficiaries are simply enjoying their new-found 'highly educated' status independently of earnings. When the sample is not split by education, the high education dummy has its most positive (statistically significant) impact for BHPS Waves 14 and beyond (since about 2004) and for those aged 45+, who represent the traditional elite. Exploring these factors remains an important topic for future research.

\section{Conclusions}

Our results contradict standard findings from growth and happiness economics, but declining LS for the older least educated, in spite of growing real income until about 2010 , is certainly consistent with the support of this group for populist movements in several countries, including Brexit in the UK. However, the obvious correlation between rising income and LS for the highly educated up to the crash leaves the insignificance of income variables in fixed-effects regressions for this group all the more surprising, and to the best of our knowledge, unprecedented in happiness economics. The older highly educated have the lowest LS for most of the period, right up to the recession, in spite of having the highest incomes and presumably the best jobs in the age group, and in contradiction to standard findings for other countries. We are left with major puzzles and an obvious need for more research in this area. 
Acknowledgements A standard disclaimer applies. Access to the British Household Panel Survey (BHPS) and Understanding Society data was obtained via the UK Data Service. Understanding Society is an initiative funded by the Economic and Social Research Council and various Government Departments, with scientific leadership by the Institute for Social and Economic Research, University of Essex, and survey delivery by NatCen Social Research and Kantar Public. Thanks are due to conference participants where earlier versions of this paper were presented (Scottish Economic Society in Perth; Work, Pensions and Labour Economics Study Group in Sheffield), and also to two anonymous referees and an Associate Editor of this journal.

\section{Compliance with ethical standards}

Conflict of interest The authors declared that they have no conflict of interests.

Ethical approval This article does not contain any studies performed by any of the authors with human participants or animals. [It uses secondary data (as per "Acknowledgements").]

Open Access This article is distributed under the terms of the Creative Commons Attribution 4.0 International License (http://creativecommons.org/licenses/by/4.0/), which permits unrestricted use, distribution, and reproduction in any medium, provided you give appropriate credit to the original author(s) and the source, provide a link to the Creative Commons license, and indicate if changes were made.

\section{Appendix 1}

See Figs. 10, 11 and 12.

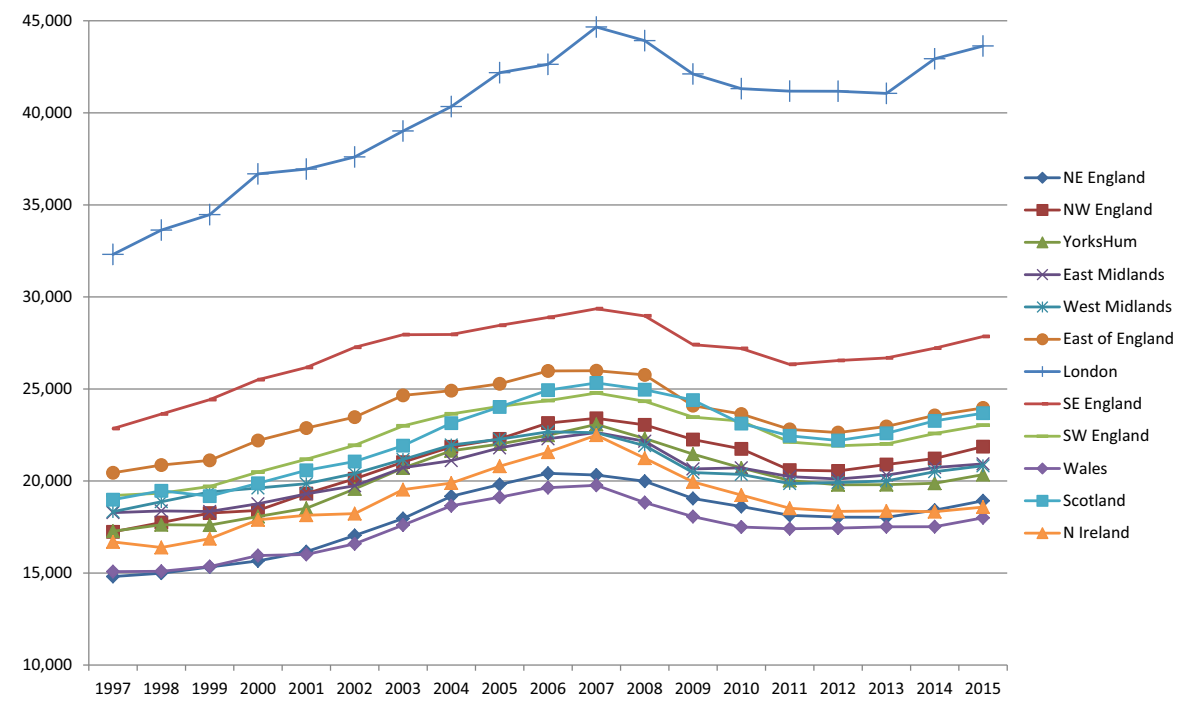

Fig. 10 Regional gross value added per capita, deflated by CPI, 1997-2015 


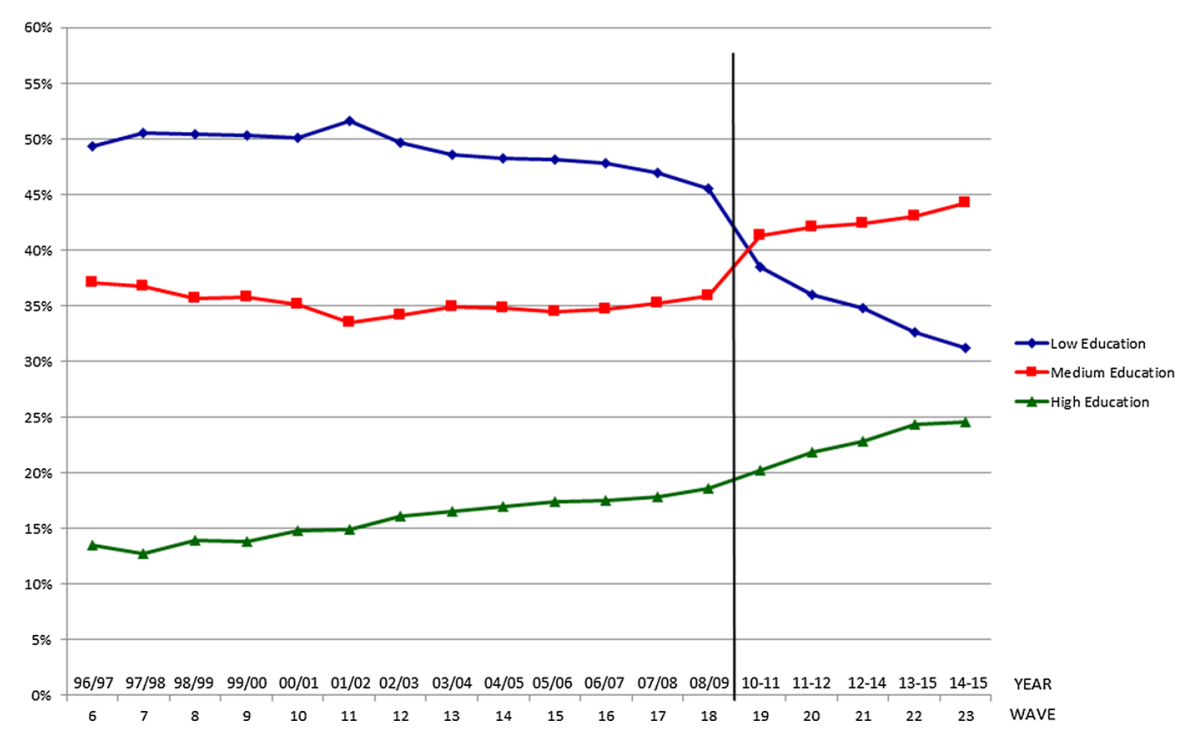

Fig. 11 Education categories, BHPS and USoc, aged $<45$, Waves 6-23

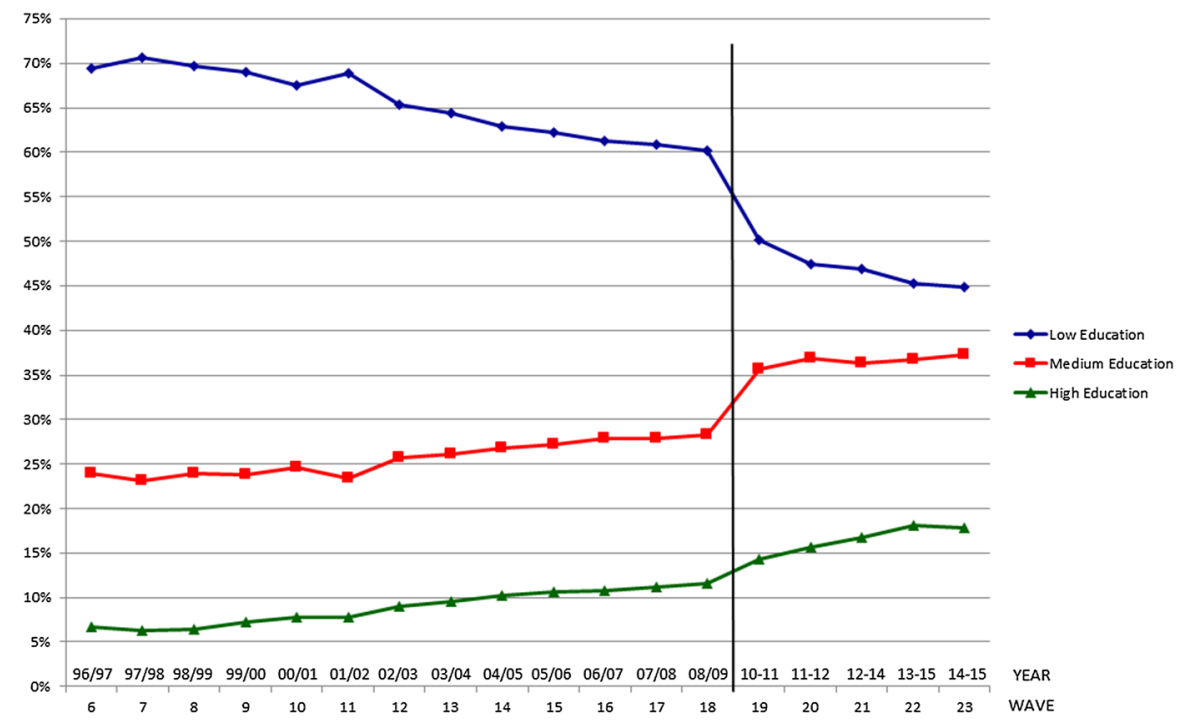

Fig. 12 Education categories, BHPS and USoc, aged 45+, Waves 6-23

\section{References}

Blundell R, Green DA, Jin W (2016) The UK wage premium puzzle: how did a large increase in university graduates leave the education premium unchanged? Institute for Fiscal Studies, working paper W16/01

Brown S, Taylor K (2014) Household finances and the 'Big Five' personality traits. J Econ Psychol 45:197-212

Brown S, Taylor K (2015) Charitable behaviour and the big five personality traits: evidence from UK panel data. IZA DP no. 9318 
Clark AE, Oswald AJ (1996) Satisfaction and comparison income. J Public Econ 61:359-381

De Neve J-E, Ward G (2017) Happiness at work, Chapter 6, Helliwell et al (2017)

De Neve J-E, Ward G, De Keulenaer F, Van Landeghem B, Kavetsos G, Norton M (2014) Individual experience of positive and negative growth is asymmetric: global evidence using subjective wellbeing data. LSE Centre for economic performance discussion paper no. 1304

Easterlin RA (1974) Does economic growth improve the human lot? In: David PA, Reder MW (eds) Nations and households in economic growth: essays in honor of Moses Abramovitz. Academic Press, New York, pp 89-125

Easterlin RA (2013) Happiness and economic growth: the evidence. IZA DP no. 7187

FitzRoy FR, Nolan MA, Steinhardt MF, Ulph D (2014) Testing the tunnel effect: comparison, age and happiness UK and German panels. IZA J Eur Labor Stud 3:24. https://doi.org/10.1186/2193-9012-324

Green F (2011) Unpacking the misery multiplier: How employability modifies the impacts of unemployment and job insecurity on life satisfaction and mental health. J Health Econ 30:265-276

Helliwell J, Layard R, Sachs J (2017) World happiness report 2017. Sustainable Development Solutions Network, New York

Kantar Public, NatCen Social Research, University of Essex, Institute for Social and Economic Research (2016) Understanding Society: Waves 1-6, 2009-2015. (data collection). 8th edn., [original data producer(s)]. UK Data Service. SN: 6614. https://doi.org/10.5255/ukda-sn-6614-9

Layard R, Mayraz G, Nickell S (2010) Does relative income matter? Are the critics right? Chapter 6. In: Diener E, Helliwell JF, Kahneman D (eds) International differences in well-being. Oxford University Press, Oxford

Moulton BR (1990) An illustration of a pitfall in estimating the effects of aggregate variables on micro units. Rev Econ Stat 72(2):334-338

Nikolaev B (2016) Does higher education increase hedonic and eudaimonic happiness. J Happiness Stud 1:2-3. https://doi.org/10.1007/s10902-016-9833-y

Nikolaev B, Rusakov P (2016) Education and happiness: an alternative hypothesis. Appl Econ Lett 23(12):827-830. https://doi.org/10.1080/13504851.2015.1111982

Pfaff T, Hirata J (2013) Testing the Easterlin hypothesis with panel data: The dynamic relationship between life satisfaction and economic growth in Germany and in the UK. Centre for Interdisciplinary Economics, working paper $4 / 2013$

Powdthavee N (2010) How much does money really matter? Estimating the causal effects of income on happiness. Empir Econ 39:77-92. https://doi.org/10.1007/s00181-009-0295-5

Powdthavee N, Lekfuangfu WN, Wooden M (2015) What's the good of education on our overall quality of life? A simultaneous equation model of education and life satisfaction for Australia. J Behav Exp Econ 54:10-21. https://doi.org/10.1016/j.socec.2014.11.002

Proto E, Rustichini A (2015) Life satisfaction, income and personality. J Econ Psychol 48:17-32

Robinson WS (1950) Ecological correlations and the behavior of individuals. Am Sociol Rev 15(3):351-357

Selvin HC (1958) Durkheim's suicide and problems of empirical research. Am J Sociol 63(6):607-619

University of Essex, Institute for Social and Economic Research (2010) British household panel survey: Waves 1-18, 1991-2009 (data collection). 7th edn., UK Data Service. SN: 5151. https://doi.org/10. 5255/ukda-sn-5151-1 